\title{
General state contrast imaging: an optimized polarimetric imaging modality insensitive to spatial intensity fluctuations
}

\author{
Guillaume Anna, ${ }^{1}$ Françcois Goudail, ${ }^{1, *}$ and Daniel Dolfi ${ }^{2}$ \\ ${ }^{1}$ Laboratoire Charles Fabry, UMR 8501, Institut d'Optique, CNRS, University Paris Sud 11, 91127 Palaiseau, France \\ ${ }^{2}$ Thales Research and Technology-France, RD128, 91767 Palaiseau Cedex, France \\ ${ }^{*}$ Corresponding author: francois.goudail@institutoptique.fr
}

Received December 13, 2011; revised January 30, 2012; accepted February 1, 2012;

posted February 2, 2012 (Doc. ID 159887); published May 17, 2012

\begin{abstract}
In active polarization imaging, one frequently needs to be insensitive to noninformative spatial intensity fluctuations. We investigate a way of solving this issue with general state contrast (GSC) imaging. It consists in acquiring two scalar polarimetric images with optimized illumination and analysis polarization states, then forming a ratio. We propose a method for maximizing the discrimination ability between a target and a background in GSC images by determining the optimal illumination and analysis states. A further advantage of this approach is to provide an objective way of quantifying the performance improvement obtained by increasing the number of degrees of freedom of a GSC imager. The efficiency of this approach is demonstrated on simulated and real-world images. () 2012 Optical Society of America
\end{abstract}

OCIS codes: $110.5405,100.0100$.

\section{INTRODUCTION}

Polarimetric images are useful for gathering information that is not visible in intensity images. They have many applications in machine vision, remote sensing, biomedical imaging, and industrial control [1-9]. One of the most frequent applications is target detection, where the goal is to optimize the contrast between a target of interest and the background. The first solutions to this problem have been obtained in the radar community $[10,11]$, where systems are intrinsically active and polarization plays an important role. Then, thanks to technological progress, several polarimetric contrast optimization strategies have been designed in the domain of optics [12-17].

One of the main issues in polarimetric target detection is robustness to noninformative spatial fluctuations, such as intensity fluctuations. Indeed, in some cases, light intensity is useful for discriminating the regions of interest, but in other instances, it is not and can create false alarms. In such cases, one would like to base detection only on purely polarimetric properties. To respond to this need, different polarimetric imaging strategies have been designed. In particular, several decompositions have been proposed to extract purely polarimetric properties from measurements [18,19]. However, the simplest way of suppressing the influence of intensity is orthogonal state contrast (OSC) imaging [20]. This technique consists in illuminating the scene with a given polarization state and in acquiring two intensity images after analyzing the light parallel and orthogonal to the illumination state. The OSC image is then obtained as the pixelwise ratio between the difference and the sum of these two images. OSC imaging is known as an efficient way to estimate the degree of polarization (DOP) of the light backscattered by the scene in certain conditions and has been used in several applications [20-23].
In this paper, we propose a generalization of OSC imaging that we call general state contrast (GSC) imaging, by allowing the illumination and analysis states of the two acquired images to vary independently, and thus to be optimized. It has to be noted that works have already been done in this direction $[\underline{13}, \underline{16}]$. It is well known that the main issue in OSC imaging is noise enhancement, due to the fact that the image is a ratio of noisy measurements $[13,24]$. This effect has to be taken into account in the criterion used for optimizing the illumination and analysis polarization states. In this paper, we will consider two commonly encountered noise sources. The first one is Poisson-distributed shot noise, since well-designed optical imaging systems using incoherent illumination are ultimately limited by this type of noise. The second one is spatial fluctuations of the polarimetric properties of the scene itself, which can be the main source of perturbation in imaging through inhomogeneously diffusive media. Furthermore, GSC imagers can have different numbers of degrees of freedom in the illumination and analysis polarization states, but a larger number of degrees of freedom generally increases complexity and cost of the imaging system. A further advantage of the proposed approach is that it defines an objective expression of the image quality in a target detection application, and thus provides a quantitative way of evaluating the improvement brought by each extra degree of freedom.

The paper is organized as follows. In Section 2 , we define GSC imaging. In Section 3, we propose an image quality criterion adapted to target detection and use it to optimize GSC imaging configurations. In Section 4 , we apply the proposed approach to the comparison of different GSC imaging configurations with different degrees of freedom. The efficiency of this method is demonstrated on simulated and real-world images. 


\section{GENERAL STATE CONTRAST IMAGING}

In this section, we recall the principle of the basic building block of any active polarization imager [25], then we present how it can be used for reducing the sensitivity to intensity fluctuations by computing the GSC image. Let us consider an active polarimetric imaging system that illuminates the scene with light whose polarization state is defined by a Stokes vector $\mathbf{S}$ and is produced by a polarization state generator (PSG) composed of two liquid crystal variable retarders associated with a linear polarizer (see Fig. 1). The polarimetric properties of a region of the scene corresponding to a pixel in the image are characterized by its Mueller matrix $M$. The Stokes vector of the light scattered by this region is $\mathbf{S}^{\prime}=M \mathbf{S}$. It is analyzed by a polarization state analyzer (PSA), which is a generalized polarizer allowing selection of photons characterized by the Stokes vector T. This PSA is composed of the same optical elements as the PSG. The number of photoelectrons measured at a pixel of the sensor is

$$
i=\frac{\eta I_{0}}{2} \mathbf{T}^{T} M \mathbf{S}
$$

where the superscript $T$ denotes matrix transposition. In this equation, $\mathbf{S}$ and $\mathbf{T}$ are unit intensity, purely polarized Stokes vectors, $I_{0}$ is a number of photons, and $\eta$ is the conversion efficiency between photons and electrons. It is thus obvious that by changing the illumination and analysis polarization states, it is possible to change the intensity coming from the objects in the scene.

It has been shown that when the polarization contains relevant information for enhancing the performance of detection of a single target over a background [26], the best strategy consists in acquiring a single scalar polarimetric image with optimal PSG and PSA states [27]. However, this acquisition method is sensitive to intensity. If this intensity is relevant for discriminating the target of interest and the background, it is valuable to use it. However, in many cases intensity fluctuations, for example, due to illumination nonuniformity, are irrelevant and can even create false alarms. In this case, it is preferable to base detection only on purely polarimetric information that is independent of the illumination. One way of reaching this objective is to perform two intensity measurements by illuminating the scene with the same linear PSG state but two orthogonal linear PSA states. One obtains

$$
i_{\|}=\frac{\eta I_{0}}{2} \mathbf{S}^{T} M \mathbf{S} \quad \text { and } \quad i_{\perp}=\frac{\eta I_{0}}{2} \mathbf{S}_{\perp}^{T} M \mathbf{S},
$$

where $M$ denotes the Mueller matrix associated with a pixel of the scene and $\mathbf{S}_{\perp}$ the Stokes vector orthogonal to $\mathbf{S}$. One then computes the OSC image in the following way:

$$
\mathrm{OSC}=\frac{i_{\|}-i_{\perp}}{i_{\|}+i_{\perp}}=\frac{\mathbf{S}^{T} M \mathbf{S}-\mathbf{S}_{\perp}^{T} M \mathbf{S}}{\mathbf{S}^{T} M \mathbf{S}+\mathbf{S}_{\perp}^{T} M \mathbf{S}}
$$

It is obvious that if the Muller matrix of the observed pixel is multiplied by a coefficient $\alpha$ that remains constant between the two sequential acquisitions of the image (which means that the intensity of the light source does not vary temporally), the OSC does not change. Moreover, it has been shown that if the Mueller matrix $M$ is a diagonal depolarizer of the form [28]

$$
M=\left[\begin{array}{llll}
1 & 0 & 0 & 0 \\
0 & a & 0 & 0 \\
0 & 0 & a & 0 \\
0 & 0 & 0 & b
\end{array}\right]
$$

then one has $\mathrm{OSC}=a$, which represents the depolarization power for linear input states [20,21]. Because of its invariance to intensity fluctuations and the fact that it estimates the depolarizing properties of objects in certain conditions of imaging, this kind of imaging system is of great interest in detection applications [21-23].

It is possible to increase the degrees of freedom in acquisition of the OSC while preserving the invariance to intensity. Indeed, we can assume that in the two acquired images, the PSG and PSA states can be anywhere on the Poincaré sphere:

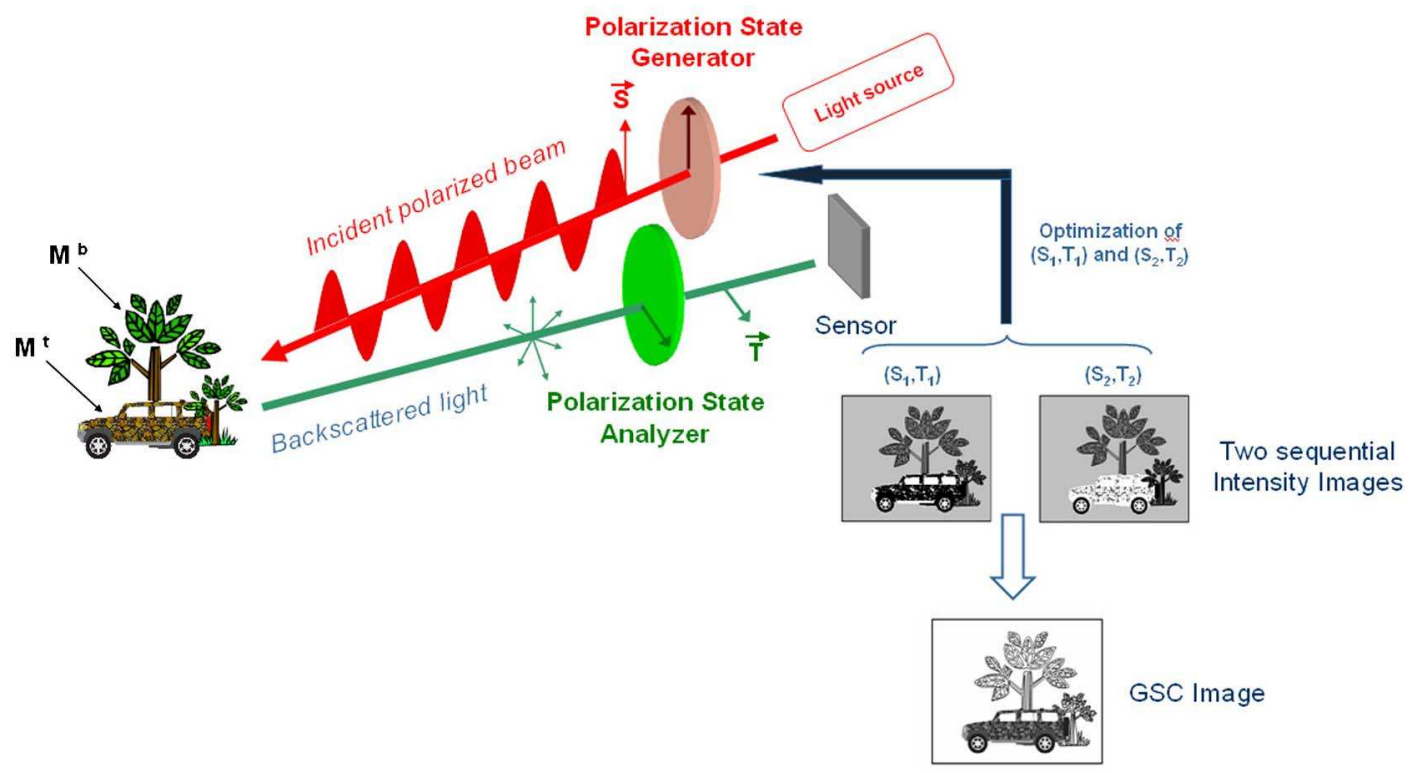

Fig. 1. (Color online) Polarimetric imaging setup. 


$$
i_{1}=\frac{\eta I_{0}}{2} \mathbf{T}_{1}^{T} M \mathbf{S}_{1} \quad \text { and } \quad i_{2}=\frac{\eta I_{0}}{2} \mathbf{T}_{2}^{T} M \mathbf{S}_{2},
$$

where $\mathbf{S}_{1}$ and $\mathbf{S}_{2}$ are the two PSG states and $\mathbf{T}_{1}$ and $\mathbf{T}_{2}$ the two PSA states. One then computes the GSC in the following way:

$$
\gamma(\theta)=\frac{i_{1}-i_{2}}{i_{1}+i_{2}}=\frac{\mathbf{T}_{1}^{T} M \mathbf{S}_{1}-\mathbf{T}_{2}^{T} M \mathbf{S}_{2}}{\mathbf{T}_{1}^{T} M \mathbf{S}_{1}+\mathbf{T}_{2}^{T} M \mathbf{S}_{2}},
$$

where we have denoted as $\theta=\left\{\mathbf{S}_{1}, \mathbf{T}_{1}, \mathbf{S}_{2}, \mathbf{T}_{2}\right\}$ the set of parameters on which the GSC depends. These parameters can be optimized to maximize some performance criterion. Of course, the adequate criterion depends on the information searched in the image. In this paper, we address the problem of target detection, and it is clear that GSC will be particularly efficient when, for example, the differences in the polarimetric properties between the target and the background are very small. In the following, we will propose a method for optimizing GSC imaging in the presence of different noise sources and demonstrate the improvement brought by this method compared to conventional OSC imaging on real-world polarimetric images.

\section{GENERALIZED STATE CONTRAST IMAGING OPTIMIZATION}

We consider an imaging system that acquires two polarimetric images with different PSG and PSA settings:

$$
i_{1}^{k}=\frac{\eta I_{0}}{2} \mathbf{T}_{1}^{T} M_{k} \mathbf{S}_{1} \quad \text { and } \quad i_{2}^{k}=\frac{\eta I_{0}}{2} \mathbf{T}_{2}^{T} M_{k} \mathbf{S}_{2},
$$

where the index $k$ denotes the pixel (for simplicity's sake, we will use in this paper one-dimensional notation for image coordinates) and $M_{k}$ denotes the Mueller matrix at pixel $k$. One then computes the GSC image as follows:

$$
\gamma_{k}(\theta)=\frac{i_{1}^{k}-i_{2}^{k}}{i_{1}^{k}+i_{2}^{k}}=\frac{\mathbf{T}_{1}^{T} M_{k} \mathbf{S}_{1}-\mathbf{T}_{2}^{T} M_{k} \mathbf{S}_{2}}{\mathbf{T}_{1}^{T} M_{k} \mathbf{S}_{1}+\mathbf{T}_{2}^{T} M_{k} \mathbf{S}_{2}} .
$$

In practice, the intensity images in Eq. (7) are perturbed by noise, and the GSC image in Eq. (8) is often too noisy to be exploited. To reduce these perturbations, one has to estimate intensity as an average over a small neighborhood $\Omega_{k}$ of $N$ pixels around pixel $k$. The GSC image is then

$$
\gamma_{k}(\theta)=\frac{\sum_{n \in \Omega_{k}} \mathbf{T}_{1}^{T} M_{n} \mathbf{S}_{1}-\sum_{n \in \Omega_{k}} \mathbf{T}_{2}^{T} M_{n} \mathbf{S}_{2}}{\sum_{n \in \Omega_{k}} \mathbf{T}_{1}^{T} M_{n} \mathbf{S}_{1}+\sum_{n \in \Omega_{k}} \mathbf{T}_{2}^{T} M_{n} \mathbf{S}_{2}} .
$$

It has been shown that in the presence of additive or shot noise, small neighborhoods of $3 \times 3 \quad(N=9)$ or $5 \times 5$ $(N=25)$ pixels significantly reduce the noise while having

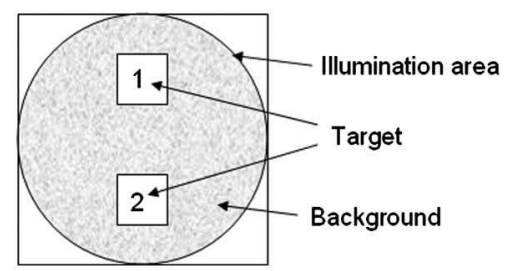

(a)

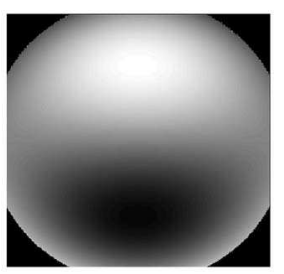

(b)
Fig. 2. (a) Scheme of the scene, (b) intensity image. a limited impact on image resolution [24,29]. It can also be noted that if full spatial resolution is necessary, the averaging can be made temporally over $N$ successive acquisitions.

Our objective in the following will be to determine the parameter set $\theta$ that optimizes the GSC image. For that purpose, one first has to choose an image quality criterion. After describing this criterion and practical ways of optimizing it, we will illustrate the efficiency of GSC in the presence of two types of noise: Poisson-distributed shot noise and spatial fluctuations of the Mueller matrix.

\section{A. Definition of the Contrast Criterion: the Bhattacharyya Distance}

We consider that the observed scene is composed of two regions: the target region $t$ and the background $b$. The objective is to have good discrimination between these two regions in the GSC image. We thus have to define a criterion characterizing the discrimination ability, and the Bhattacharyya distance has been shown to be an efficient way of doing this [30]. The Bhattacharyya distance is defined as follows. Let us consider two probability density functions (PDFs) $P_{t}(x)$ and $P_{b}(x)$. In our case, these PDFs correspond to the noise statistics of the pixels in regions $t$ (target) and $b$ (background). The Bhattacharyya distance between these two sets is defined as

$$
\mathcal{B}=-\log \left[\int_{\mathcal{D}}\left[P_{t}(x) P_{b}(x)\right]^{1 / 2} \mathrm{~d} x\right],
$$

with $\mathcal{D}$ the definition domain of $P^{a}$ and $P^{b}$. The Bhattacharyya distance is a scalar value that quantifies the similarity between the PDFs $P_{t}(x)$ and $P_{b}(x)$. It belongs to the interval $[0 ;+\infty[$. It is equal to zero when the PDFs are identical and infinite when the PDF supports do not overlap. We have to note that in the following, the Bhattacharrya distance will be applied to GSC images, and the variable $x$ in Eq. (10) will be related to the value of the GSC at each pixel of the sensor.

To be efficient, this criterion has to take into account the relevant sources of fluctuations in the image. The intensity images in Eq. (7) can be affected by different sources of perturbations, such as additive sensor noise, Poisson-distributed shot noise, and spatial fluctuations of the polarimetric properties in the scene. These perturbations result in fluctuations of the GSC image, whose statistics are difficult to express in closed form. For simplicity's sake, we will assume that the PDFs of the GSC in the two regions are Gaussian, defined by their mean values $\langle\gamma(\theta)\rangle_{u}$, where $u=\{t, b\}$ denotes the region, and their variances $\sigma_{u}^{2}(\theta)$. This is clearly an approximation, but it has been shown that in the presence of low level additive Gaussian and Poisson noise, the PDF of the GSC is close to a Gaussian $[24,29]$. Moreover, it will be observed in simulated and real images [see Figs. 3(b), 4(b), and 7] that the histograms of regions of interest are indeed close to Gaussian curves. Within this hypothesis, the Bhattacharyya distance has the following expression:

$$
\mathcal{B}(\theta)=\frac{1}{4} \frac{\left[\langle\gamma(\theta)\rangle_{t}-\langle\gamma(\theta)\rangle_{b}\right]^{2}}{\sigma_{t}^{2}(\theta)+\sigma_{b}^{2}(\theta)}+\frac{1}{2} \log \left[\frac{1}{2}\left(\frac{\sigma_{t}(\theta)}{\sigma_{b}(\theta)}+\frac{\sigma_{b}(\theta)}{\sigma_{t}(\theta)}\right)\right],
$$

with $\theta=\left\{\mathbf{S}_{1}, \mathbf{T}_{1}, \mathbf{S}_{2}, \mathbf{T}_{2}\right\}$. The higher the value of $\mathcal{B}(\theta)$, the easier will be the discrimination of the target from the 


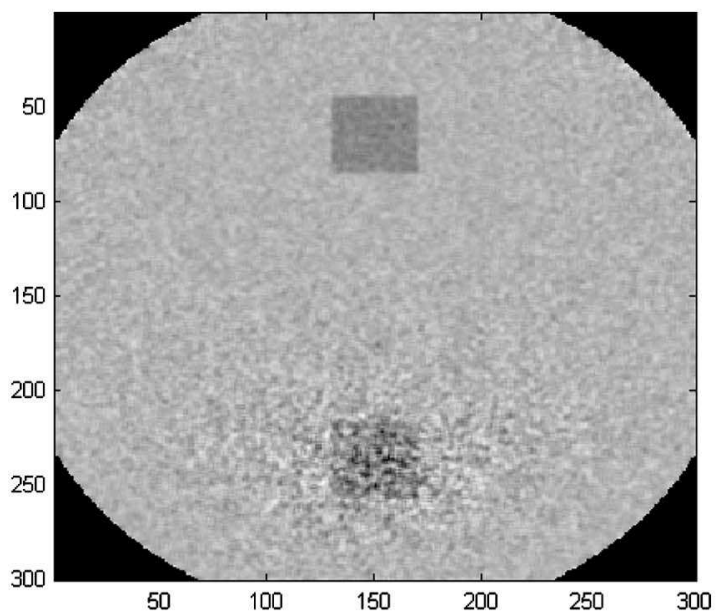

(a)

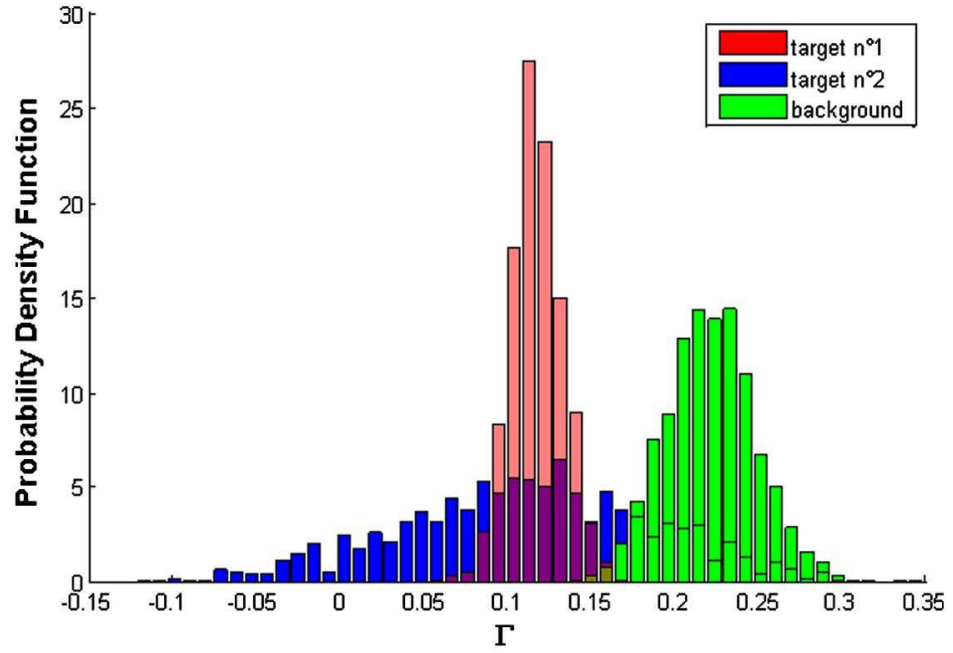

(b)

Fig. 3. (Color online) (a) GSC image of the scene in Fig. 2 after optimization of $\mathcal{D}(\theta)$, (b) PDF of the targets and of the background.

background. Our goal is now to compute the optimal set of parameters $\theta$ that maximizes this criterion.

\section{B. Computational Issue for the Optimization}

To perform the search of the set $\theta$ that maximizes the Bhattacharyya distance defined in Eq. (11), one has to take into account the number of parameters that have to be optimized simultaneously. Indeed, each intensity image depends on four parameters, e.g., the azimuth and ellipticity of the PSG and PSA states. Optimizing the two intensity images needed for GSC calculation thus involves eight parameters, which is quite large, and it is thus likely that the performance criterion will have local maxima. It is thus necessary to use an algorithm robust to the presence of local maxima. After comparing different solutions, we have chosen the shuffled complex evolution (SCE-UA) method [31]. This algorithm consists in generating different sets of illumination and analysis polarization states and in changing them by using a global evolution framework to finally converge to a parameter set that leads to the highest value of the Bhattacharyya distance. We have verified that in our applications, it converges rapidly to the global maximum researched.

\section{Optimization in the Presence of Nonuniform Illumination Intensity}

Our purpose in this section is to study the influence of the signal to noise ratio of the intensity images involved in GSC computation on the choice of the optimal PSG and PSA states. We will assume that the illumination intensity is nonuniform on the scene and, to simplify the discussion, that the dominant source of perturbation is Poisson-distributed shot noise. An approximate closed-form expression of the variance of the GSC in the presence of Poisson noise has been determined in [29]

$$
\sigma^{2}=\frac{\left(1-\gamma^{2}\right)}{N \times i_{\text {tot }}}
$$

where $\gamma$ is the true value of the GSC, $N$ the size of the neighborhood on which the GSC is estimated [see Eq. (9)], and $i_{\text {tot }}$ the sum of the true values of the two intensity measurements defined in Eq. ()):

$$
i_{\mathrm{tot}}=i_{1}+i_{2}
$$

It is interesting to notice on Eq. (12) that even if the true value $\gamma$ of the GSC does not depend on the intensity (this is precisely the reason for the choice of this value), that is not the case for its variance $\sigma^{2}$. Indeed, the higher the $i_{\text {tot }}$, the lower is this variance. This phenomenon can be explained by the fact that the signal to noise ratio in the intensity measurements increases as $\sqrt{i_{\text {tot }}}$, which makes the estimation of $\gamma$ easier. This observation is critical in the presence of strong nonuniform illumination. Indeed, it can be preferable to have a lower difference of GSC mean values between the two regions $b$ and $t$ with few fluctuations than a higher difference with too strong fluctuations.

Let us first illustrate this observation on a simulated image. The scheme of the scene is presented in Fig. 2(a). It is composed of two square pieces of the same material, which constitute the target $(t)$, over a background $(b)$. The average Mueller matrices of the target and the background are given in Table 1. For illustration purposes, we have chosen for the target and the background simple diagonal depolarizer matrices, which constitute special members of the vast class of depolarizing Mueller matrices [28]. We simulate a strongly nonuniform illumination having smooth variations. The image that would be observed with a standard intensity imager is represented in Fig. 2(b). We can observe the spatial variation of the illumination, and we can notice that since the target and the background have the same intensity reflectivities, they cannot be discriminated on this image.

Table 1. Average Mueller Matrices of the Target $(t)$ and the Background $(b)$

\begin{tabular}{c}
\hline$M^{b}$ \\
\hline$\left[\begin{array}{cccc}1 & 0 & 0 & 0 \\
0 & 0.80 & 0 & 0 \\
0 & 0 & 0.84 & 0 \\
0 & 0 & 0 & 0.60\end{array}\right] \quad\left[\begin{array}{cccc}1 & 0 & 0 & 0 \\
0 & 0.80 & 0 & 0 \\
0 & 0 & 0.87 & 0 \\
0 & 0 & 0 & 0.65\end{array}\right]$ \\
\hline
\end{tabular}


Table 2. Optimal States Maximizing the Discrimination Criteria $\mathcal{D}(\theta)$ [see Eq. (14)] and $\mathcal{B}(\theta)$

[see Eq. (11)] ${ }^{a}$

\begin{tabular}{lllll}
\hline Criterion & $\mathbf{S}_{1}$ & $\mathbf{T}_{1}$ & $\mathbf{S}_{2}$ & $\mathbf{T}_{2}$ \\
\hline $\mathcal{D}(\theta)$ & $\left(-90^{\circ}, 0^{\circ}\right)$ & $\left(0^{\circ}, 0^{\circ}\right)$ & $\left(-45^{\circ}, 0^{\circ}\right)$ & $\left(45^{\circ}, 0^{\circ}\right)$ \\
$\mathcal{B}(\theta)$ & $\left(0^{\circ}, 45^{\circ}\right)$ & $\left(0^{\circ},-45^{\circ}\right)$ & $\left(0^{\circ}, 45^{\circ}\right)$ & $\left(0^{\circ}, 45^{\circ}\right)$ \\
\hline${ }^{a}$ States are represented as $(\alpha, \varepsilon)$, where $\alpha$ is the & azimuth and $\varepsilon$ the ellipticity.
\end{tabular}

In order to illustrate the influence of illumination intensity on noise in the GSC image, we first compute the parameter set $\theta$ that maximizes the following criterion:

$$
\mathcal{D}(\theta)=\left[\langle\gamma(\theta)\rangle_{t}-\langle\gamma(\theta)\rangle_{b}\right]^{2}
$$

This criterion is simply a squared difference between the mean values; it does not take into account the variation of the noise variance with $\theta$. The pair of PSA and PSG states that maximizes this criterion is given in Table 2 . We can note that these optimal states do not correspond to the classic OSC, since the two illumination states are different and the illumination and analysis states are orthogonal for each acquired image. We have represented the optimal GSC image in Fig. 3(a) and the estimated PDFs of the two targets and of the background in the dark (bottom) part of the scene in Fig. 3(b). We can see that the average values $\langle\gamma\rangle_{t}$ are the same on the two targets, but the variances are different. The variance in the dark part of the scene is much larger than that in the bright region. This result is in agreement with the expression of the variance in Eq. (12), which shows that the variance is inversely proportional to $i_{\mathrm{av}}$. The difference of average values between the background and the target is about 0.12 , but the overlap between the PDF of target no. 2 and the background is also very large, which will lead to detection errors.

Let us now take into account the influence of the noise by optimizing the Bhattacharyya distance defined in Eq. (11), with $\sigma_{u}^{2}(\theta)$ expressed in Eq. (12). The optimization is done in the dark (bottom) part of the image, because it is there that the separability is the most difficult to obtain. The pair of PSG and PSA states maximizing this criterion is given in Table 2
They are circular and thus different from those obtained with criterion $\mathcal{D}(\theta)$. They thus lead to different PDFs for the targets and the background. The estimated PDFs of the two targets and of the background in the dark part of the scene are presented in Fig. 4(b) together with the optimal GSC image in Fig. 4(a). The difference in average values between the background and the target is now only 0.05 , that is twice less than in the previous case. However, the discrimination ability is significantly better, since the overlap between the histograms of target no. 2 and of the background is much reduced.

Let us now illustrate this conclusion in a real-world example. The background is composed of white paper, and the target is a piece of translucent adhesive, so the polarimetric properties of the target and the background are very similar. The scheme of the scene and the intensity image are given in Fig. 5. We can see that the target cannot be discriminated from the background on a standard intensity image. The GSC image obtained after maximizing $\mathcal{D}(\theta)$ is presented in Fig. 6(a) and that obtained by maximizing $\mathcal{B}(\theta)$ in Fig. $6(\mathrm{~b})$. We observe that it is easier to detect the target on Fig. 6(b), which corresponds to an optimization taking into account the dependence of noise variance with $\theta$. We have represented in Fig. 7 the estimated PDFs of the target and the background in these two images. We can observe that, as in the simulated example, the difference between the average values $\langle\gamma\rangle_{t}$ and $\langle\gamma\rangle_{b}$ is higher when we optimize $\mathcal{D}(\theta)$, but the overlap between their PDFs is high. This overlap is reduced when $\mathcal{B}(\theta)$ is optimized, even if the difference of average values has decreased. The conclusions are in agreement with those presented in the simulated example: optimizing the Bhattacharyya distance enables us to find a better compromise between average class separation and noise variance.

In this section, we have analyzed the influence of Poisson noise on GSC optimization. If the illumination intensity is low or varies strongly, the variation of noise variance with the PSG and PSA states must be taken into account, and the Bhattacharyya distance is an efficient way of doing this. Furthermore, the optimization of the GSC image has to be done between the target and the background that are in the darkest parts of the scene to increase the global discrimination ability, since this corresponds to the most difficult

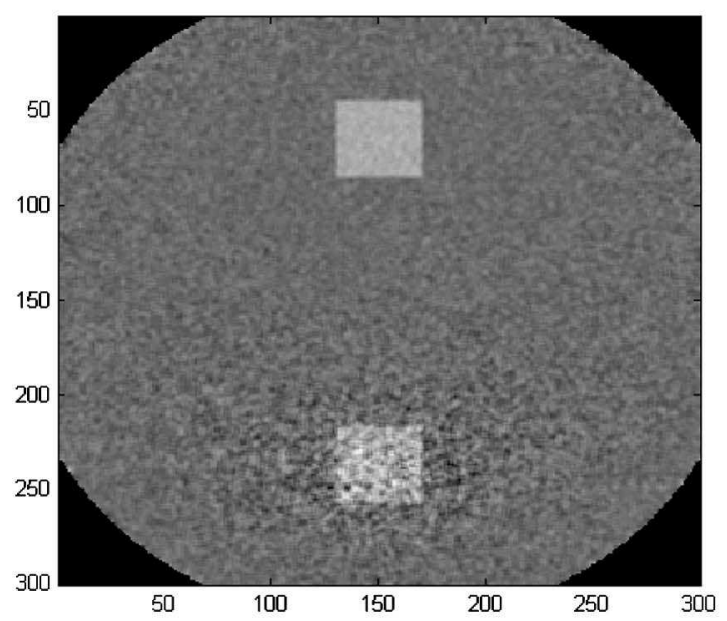

(a)

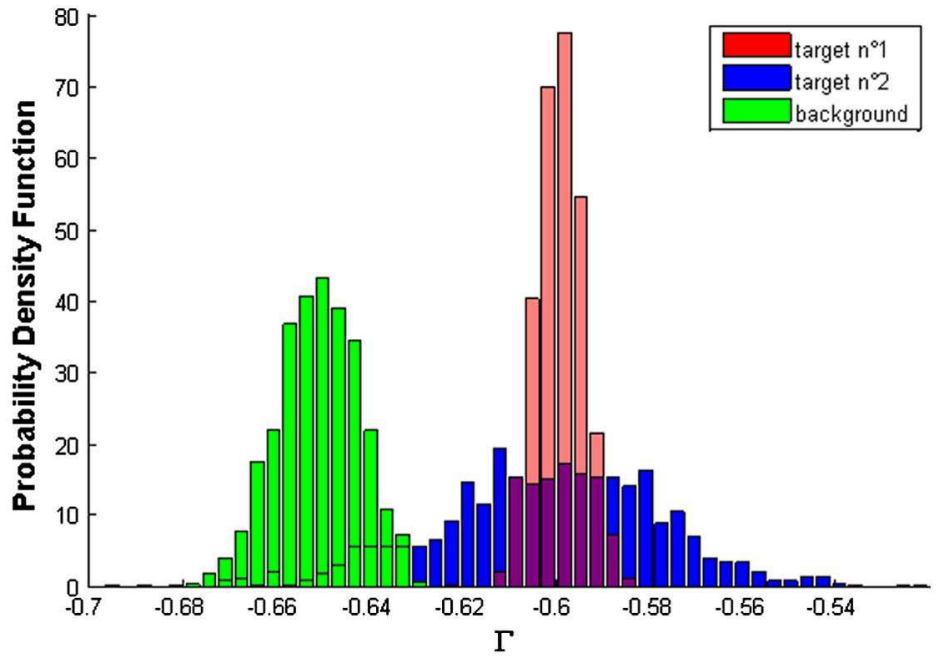

(b)

Fig. 4. (Color online) (a) GSC image of the scene in Fig. $\underline{2}$ after optimization of $\mathcal{D}(\theta)$, (b) PDFs of the targets and of the background. 


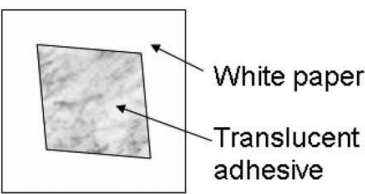

(a)

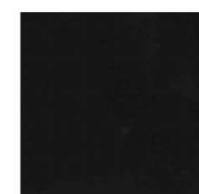

(b)
Fig. 5. (a) Schema of the scene, (b) intensity image.

case. Finally, it can be noted that the same conclusions would be reached if additive noise was the dominant source of perturbations in intensity images; an approximate expression of the variance on the GSC induced by additive noise can be found in [29].

\section{Optimization in the Presence of Spatial Fluctuations of the Polarimetric Properties in the Scene}

Let us now consider that the main source of noise disturbing the image is not linked to detection but to the spatial fluctuations of the polarimetric properties of the scene. In this case also, it is beneficial to take into account the dependence of the noise variance on PSG and PSA states. Let us illustrate this effect in a real-world example. The scene is composed of a piece of adhesive tape [the target $(\mathrm{t})$ ] over a polarizer [the background (b)], and both are placed behind translucent diffusing papers and a translucent foam-like plastic sheet with bubbles. It is impossible to discriminate the adhesive tape on the standard intensity image as presented in Fig. 8(a). We first optimize the squared difference of average values $\mathcal{D}(\theta)$ [see Eq. (14)]. The optimal set of parameters is given in Table 3, and the corresponding GSC image is presented in Fig. 9 (b). In this image, the target can hardly be discriminated due to the presence of high fluctuations.

Let us now take into account these fluctuations by optimizing the Bhattacharyya distance in Eq. (11). Contrary to the case where only additive or Poisson noises are present, we could not find any closed-form expression of the variance of the GSC in the presence of Mueller matrix fluctuations. We thus choose to estimate the variances $\sigma_{u}^{2}(\theta)$ from training data corresponding to the two regions of the scene. Letting $\Omega_{u}$ be a set of $N_{u}$ pixels representative of region $u \in\{b, t\}$, the value of the variance in region $u$ of the GSC image is estimated as

$$
\sigma_{u}^{2}(\theta)=\frac{1}{N_{u}} \sum_{k \in \Omega_{u}}\left(\gamma_{k}(\theta)-\overline{\gamma_{u}}(\theta)\right)^{2},
$$

with

$$
\overline{\gamma_{u}}(\theta)=\frac{1}{N_{u}} \sum_{k \in \Omega_{u}} \gamma_{k}(\theta) .
$$

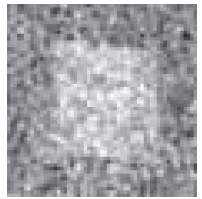

$\mathcal{B}=0.76$

(a)

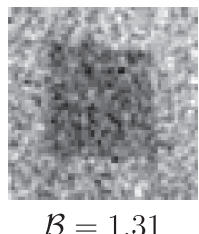

(b)
Fig. 6. GSC images of the scene in Fig. 5 after optimization of (a) $\mathcal{D}(\theta)$, (b) $\mathcal{B}(\theta)$.
It can be seen in Table 3 that the set of PSG and PSA states maximizing the Bhattacharyya distance is different from that maximizing the squared difference of average values. We can notice that they are elliptic and nontrivial. The final GSC image is also different (see Fig. 9). It is now much easier to discriminate the target from the background since the variance of the fluctuations in the image has been reduced. As a conclusion, optimization of the PSG and PSA states can lead to significant improvement of discrimination ability in GSC images. To obtain this result, it is necessary to use an optimization criterion that takes into account the variation of the noise variance as a function of the PSG and PSA states.

\section{CHOOSING THE NUMBER OF DEGREES OF FREEDOM OF A GSC IMAGER}

In the previous section, we have assumed that in the imaging system of Fig. 1, the polarization states of the PSG and the PSA could vary on the whole Poincaré sphere. This can be realized experimentally with liquid crystal variable retarders. However, this equipment is costly, and in many cases, it would be preferable to use simpler systems, which can be cheaper and easier to implement. Of course, these simpler systems have to keep a sufficient level of performance. One of the advantages of the optimization approach proposed in the previous section is that it provides a way to objectively compare the performance of different GSC acquisition setups with different numbers of degrees of freedom. We will illustrate this capacity in a real-world imaging example.

Let us consider the five different imaging configurations summarized in Table 4 . In the first four configurations, the PSG states are identical for the two acquired images $\left(\mathbf{S}_{1}=\mathbf{S}_{2}\right)$ and the two PSA states are orthogonal $\left(\mathbf{T}_{1} \perp \mathbf{T}_{2}\right)$. These two properties make it possible to use a setup using a Wollaston prism that simultaneously acquires two images with orthogonal PSA states on two different parts of an imaging sensor [23]. The two intensity images are thus acquired simultaneously, which is important for visualizing a rapidly evolving scene.

The difference among the first four configurations is thus only the number of degrees of freedom available to optimize the discrimination ability (i.e., the size of the set $\theta$ ). In configuration no. 1 , the PSG state is linear with an azimuth of $0^{\circ}$ and the PSA states are linear and orthogonal with azimuths of $0^{\circ}$ and $90^{\circ}$ : no degrees of freedom are available. In configuration no. 2, the PSG has 1 degree of freedom: its azimuth can vary, while the PSA states are parallel and orthogonal to it. In configuration no. 3, both the azimuth and the ellipticity of the PSG can be optimized, which increases the number of degrees of freedom to 2. Finally, in configuration no. 4, the PSG and PSA states are decoupled: their azimuth and ellipticities can vary independently, while the two PSA states remain orthogonal. There are thus 4 degrees of freedom. These four imaging configurations can be implemented with the setup based on a Wollaston prism described in [23]. One may wonder whether there is an interest in relaxing the constraints due to this architecture in order to further increase the discrimination ability. To answer this question, we consider configuration no. 5, in which the PSG and PSA states for each intensity image can be optimized separately and lie anywhere on the Poincaré sphere, which amounts to having 8 degrees of freedom. 


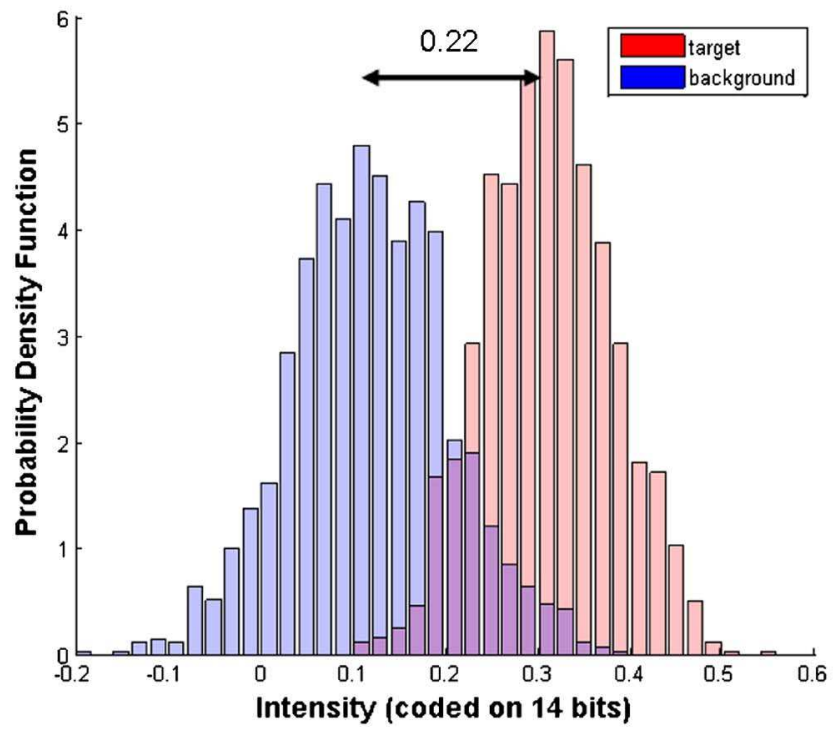

(a)

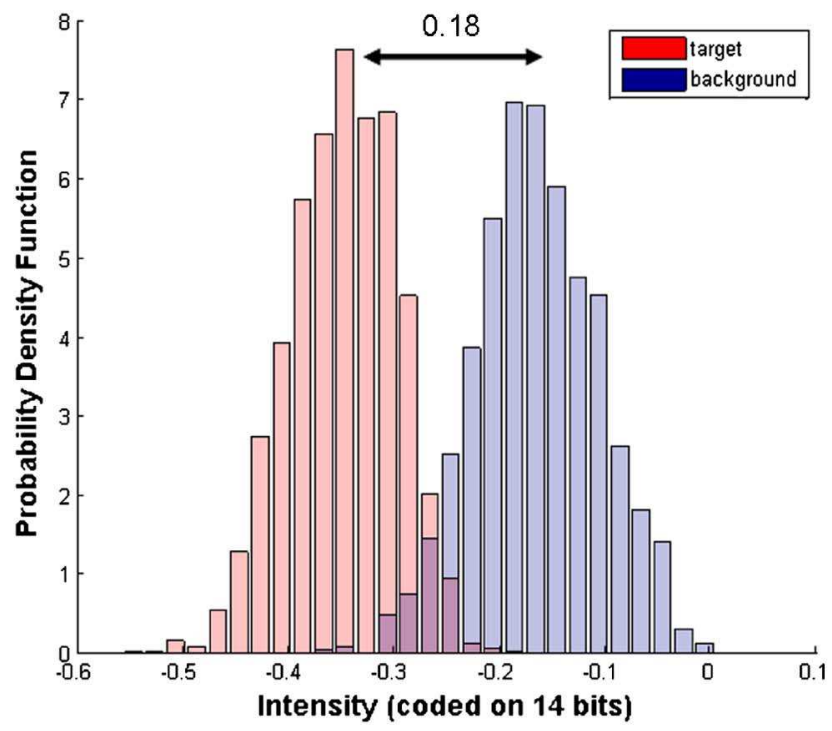

(b)

Fig. 7. (Color online) Estimated PDFs of the target and the background in the images of Fig. $\underline{6}$, obtained after optimization of (a) $\mathcal{D}(\theta)$, (b) $\mathcal{B}(\theta)$.

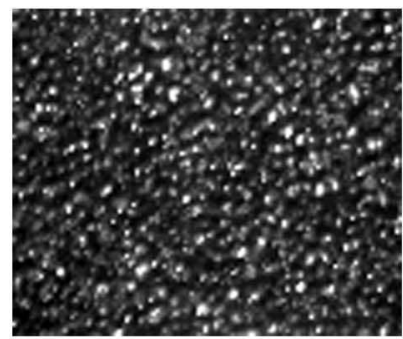

(a)

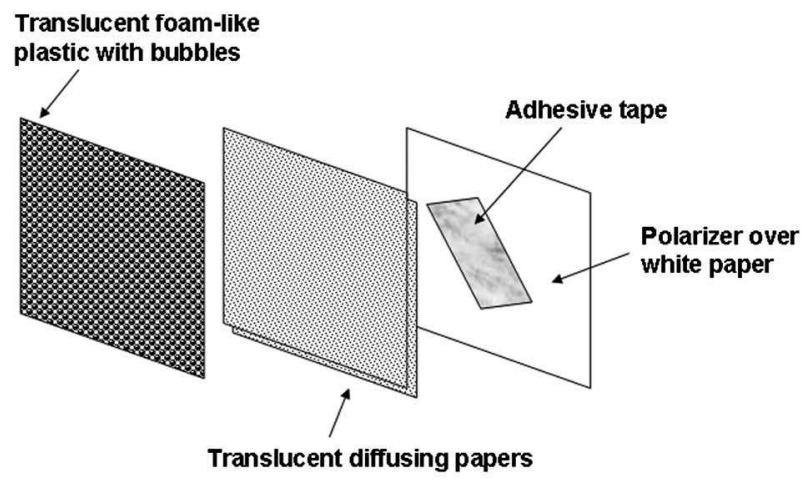

(b)

Fig. 8. (a) Intensity image of the scene, (b) scheme of the scene.

Let us now consider a scene composed of three pieces of translucent adhesive tape-which will constitute the targets_-over a background composed of a polarizer over

Table 3. Optimal States Maximizing the Discrimination Criteria $\mathcal{D}(\theta)$ [see Eq. (14)] and $\mathcal{B}(\theta)$ [see Eq. (11)]

\begin{tabular}{lllll}
\hline$(\alpha, \varepsilon)$ & $\mathbf{S}_{1}$ & $\mathbf{T}_{1}$ & $\mathbf{S}_{2}$ & $\mathbf{T}_{2}$ \\
\hline Without noise & $\left(5^{\circ},-21^{\circ}\right)$ & $\left(-60^{\circ},-4^{\circ}\right)$ & $\left(28^{\circ},-11^{\circ}\right)$ & $\left(45^{\circ},-6^{\circ}\right)$ \\
With noise & $\left(11^{\circ},-8^{\circ}\right)$ & $\left(37^{\circ}, 15^{\circ}\right)$ & $\left(31^{\circ}, 6^{\circ}\right)$ & $\left(-80^{\circ}, 7^{\circ}\right)$ \\
\hline
\end{tabular}

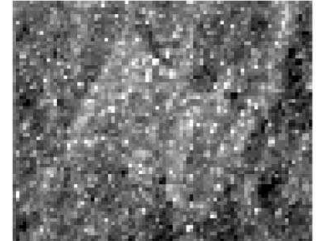

(a)

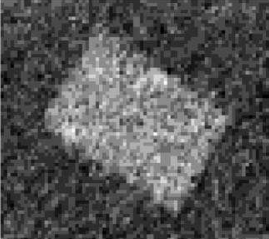

(b)
Fig. 9. GSC images of the scene in Fig. $\underline{8}$ after optimization of (a) $\mathcal{D}(\theta)$, (b) $\mathcal{B}(\theta)$ white paper. Both are placed behind a nonuniform birefringent scattering medium. The scheme of the scene and its standard intensity image are given in Fig. 10. We can see that in this image, the targets are not discriminated from the background.

Table 4. GSC Imaging Configurations with Different Degrees of Freedom ${ }^{a}$

\begin{tabular}{|c|c|c|c|c|c|}
\hline Configuration & $\begin{array}{l}\text { Degrees } \\
\text { of Freedom }\end{array}$ & $\mathbf{S}_{1}$ & $\mathbf{T}_{1}$ & $\mathbf{S}_{2}$ & $\mathbf{T}_{2}$ \\
\hline No. 1 & 0 & $\begin{array}{l}\alpha \text { fixed } \\
\varepsilon \text { fixed }\end{array}$ & $\mathbf{S}_{1}$ & $\mathbf{S}_{1}$ & $\mathbf{S}_{1}^{\perp}$ \\
\hline No. 2 & 1 & $\begin{array}{l}\alpha \text { varies } \\
\varepsilon \text { fixed }\end{array}$ & $\mathbf{S}_{1}$ & $\mathbf{S}_{1}$ & $\mathbf{S}_{1}^{\perp}$ \\
\hline No. 3 & 2 & $\begin{array}{l}\alpha \text { varies } \\
\varepsilon \text { varies }\end{array}$ & $\mathbf{S}_{1}$ & $\mathbf{S}_{1}$ & $\mathbf{S}_{1}^{\perp}$ \\
\hline No. 4 & 4 & $\begin{array}{l}\alpha_{1} \text { varies } \\
\varepsilon_{1} \text { varies }\end{array}$ & $\begin{array}{l}\alpha_{2} \text { varies } \\
\varepsilon_{2} \text { varies }\end{array}$ & $\mathbf{S}_{1}$ & $\mathbf{T}_{1}^{\perp}$ \\
\hline No. 5 & 8 & $\left(\alpha_{1}, \varepsilon_{1}\right)$ & $\left(\alpha_{2}, \varepsilon_{2}\right)$ & $\left(\alpha_{3}, \varepsilon_{3}\right)$ & $\left(\alpha_{4}, \varepsilon_{4}\right)$ \\
\hline
\end{tabular}

${ }^{a}(\alpha, \varepsilon)$ denotes the azimuth and the ellipticity of the polarization states, and $\perp$ denotes the orthogonality of a polarization state with another. 


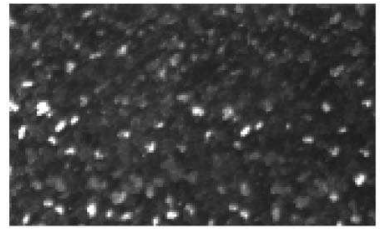

(a)

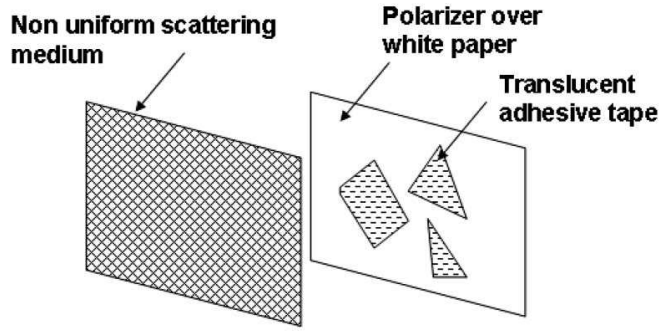

(b)

Fig. 10. (a) Intensity image, (b) scheme of the scene.

$\begin{array}{ccc}\text { Configuration } & \mathcal{B} & \text { DOP } / \text { GSC image } \\ n^{\circ} 1 & \mathcal{B}_{\text {theo }}=0.01 / \mathcal{B}_{\text {exp }}=0.16 \\ n^{o} 2 & \mathcal{B}_{\text {theo }}=3.45 / \mathcal{B}_{\text {exp }}=3.42 \\ n^{\circ} 3 & \mathcal{B}_{\text {theo }}=4.92 / \mathcal{B}_{\text {exp }}=4.11 \\ n^{\circ} 4 & \mathcal{B}_{\text {theo }}=7.7 / \mathcal{B}_{\text {exp }}=6.8 \\ n^{\circ} 5 & \mathcal{B}_{\text {theo }}=27.25 / \mathcal{B}_{\text {exp }}=18.9\end{array}$

Fig. 11. Optimal GSC images in the different configurations presented in Section 4 . $\mathcal{B}_{\text {theo }}$ and $\mathcal{B}_{\exp }$ denote, respectively, the Bhattacharyya distance computed from the database and the real images.

Table 5. Optimal States for the GSC Images in the Different Configurations Presented in Table 4

\begin{tabular}{lllll}
\hline Configuration & $\mathbf{S}_{1}$ & $\mathbf{T}_{1}$ & $\mathbf{S}_{2}$ & $\mathbf{T}_{2}$ \\
\hline No. 1 & $\left(0^{\circ}, 0^{\circ}\right)$ & $\left(0^{\circ}, 0^{\circ}\right)$ & $\left(0^{\circ}, 0^{\circ}\right)$ & $\left(90^{\circ}, 0^{\circ}\right)$ \\
No. 2 & $\left(-69^{\circ}, 0^{\circ}\right)$ & $\left(-69^{\circ}, 0^{\circ}\right)$ & $\left(-69^{\circ}, 0^{\circ}\right)$ & $\left(21^{\circ}, 0^{\circ}\right)$ \\
No. 3 & $\left(-90^{\circ},-39^{\circ}\right)$ & $\left(-90^{\circ},-39^{\circ}\right)$ & $\left(-90^{\circ},-39^{\circ}\right)$ & $\left(0^{\circ}, 39^{\circ}\right)$ \\
No. 4 & $\left(64^{\circ},-7^{\circ}\right)$ & $\left(-20^{\circ},-32^{\circ}\right)$ & $\left(64^{\circ},-7^{\circ}\right)$ & $\left(70^{\circ}, 32^{\circ}\right)$ \\
No. 5 & $\left(49^{\circ},-38^{\circ}\right)$ & $\left(79^{\circ},-32^{\circ}\right)$ & $\left(54^{\circ}, 18^{\circ}\right)$ & $\left(57^{\circ}, 15^{\circ}\right)$ \\
\hline
\end{tabular}

To compute the optimal states maximizing the contrast in the five considered configurations, we use a database containing sets of Mueller matrices associated with the targets and the background. The optimal images obtained using the different imaging configurations are presented, together with the values of the Bhattacharyya distance, in Fig. 11, and the corresponding optimal pairs of PSG/PSA states are presented in Table 5 . We can see that by increasing the number of degrees of freedom, the discrimination ability is significantly enhanced. Indeed, configuration no. 1 yields a poor contrast.
By allowing the azimuth of the PSG to vary in configuration no. 2, we observe that the azimuth of the optimal PSG state is quite different from that used in configuration no. 1 . The gain in discrimination ability is significant. If we now use the possibility of optimizing the ellipticity of the PSG and PSA in system no. 3, we observe that the optimal states are close to circular, which means that some information relevant to discrimination is contained in this polarization state. However, there remain strong fluctuations. In system no. 4, the PSG and the PSA are optimized separately, which corresponds to 4 degrees of freedom. We observe that these new degrees of freedom enable us to significantly improve the discrimination ability with respect to system no. 3. It is also interesting to note that the optimal PSG states are close to those obtained with system no. 2, while the PSA states are close to those obtained with system no. 3. Finally, in configuration no. 5, where all the constraints on PSG and PSA are relaxed, we observe that the optimal states are different from those obtained with the other configurations, and the value of the Bhattacharyya distance $(\mathcal{B}=18.9)$ is significantly larger than those obtained before. The targets are much more easily discriminated from the background, and the fluctuations are reduced. This example allows us to conclude that in certain applications, the full use of GSC degrees of freedom can help increase the discrimination ability significantly.

\section{CONCLUSION}

GSC imaging, as a polarimetric imaging modality, can reveal contrasts that do not appear in standard intensity images. It has the further advantage of being insensitive to intensity fluctuations. Its drawback is that since it is a ratio of noisy data, noise is enhanced in regions with low intensity values. Taking this fact into account, we have proposed a method for maximizing the discrimination ability between a target of interest and a background by optimizing the illumination and analysis polarization states. This method makes it possible to determine the best compromise between class separation and minimization of the fluctuations in the GSC image.

One of the advantages of the proposed approach is that it allows one to perform an objective comparison of the performance of GSC configurations with different degrees of freedom. It is thus possible to evaluate the added value of each degree of freedom in a given application. We have demonstrated the efficiency of this approach in an application example, where making use of the full set of 8 degrees of freedom brings significant improvement in discrimination ability. Of course, in practice, this gain has to be weighed against the increasing cost and complexity usually associated with increasing the number of degrees of freedom. However, the 
approach we propose provides objective elements for this choice.

This work has many perspectives. It would be interesting to generalize the method to the discrimination of more than two regions, as was recently done for scalar polarimetric images [32], and to investigate adaptive optimization strategies that would not require prior knowledge about the statistics of the regions to discriminate.

\section{ACKNOWLEDGMENTS}

The authors thank R. Horisaki for fruitful discussions and the anonymous reviewers for their comments, which were very helpful in improving the quality of this paper. G. Anna's Ph.D. thesis is supported by the Délégation Générale pour l'Armement, MRIS domain IMAT.

\section{REFERENCES}

1. J. E. Solomon, "Polarization imaging," Appl. Opt. 20, 1537-1544 (1981).

2. R. Walraven, "Polarization imagery," Opt. Eng. 20, 14-18 (1981).

3. J. S. Tyo, M. P. Rowe, E. N. Pugh, and N. Engheta, "Target detection in optical scattering media by polarization-difference imaging," Appl. Opt. 35, 1855-1870 (1996).

4. L. B. Wolff, "Polarization vision: a new sensory approach to image understanding," Image Vis. Comput. 15, 81-93 (1997).

5. P. J. Wu, J. Joseph, and T. Walsh, "Stokes polarimetry imaging of rat tail tissue in a turbid medium: degree of linear polarization image maps using incident linearly polarized light," J. Biomed. Opt. 11, 014031 (2006).

6. J. S. Tyo, D. L. Goldstein, D. B. Chenault, and J. A. Shaw, "Review of passive imaging polarimetry for remote sensing applications," Appl. Opt. 45, 5453-5469 (2006).

7. M. Alouini, F. Goudail, A. Grisard, J. Bourderionnet, D. Dolfi, I. Baarstad, T. Løke, P. Kaspersen, and X. Normandin, "Active polarimetric and multispectral laboratory demonstrator: contrast enhancement for target detection," Proc. SPIE 6396, 63960B (2006).

8. J. M. Bueno, J. Hunter, C. Cookson, M. Kisilak, and M. Campbell, "Improved scanning laser fundus imaging using polarimetry," J. Opt. Soc. Am. A 24, 1337-1348 (2007).

9. P. Terrier, V. Devlaminck, and J. M. Charbois, "Segmentation of rough surfaces using a polarization imaging system," J. Opt. Soc. Am. A 25, 423-430 (2008)

10. A. B. Kostinski and W. M. Boerner, "On the polarimetric contrast optimization," IEEE Trans. Antennas Propag. 35, 988-991 (1987).

11. A. A. Swartz, H. A. Yueh, J. A. Kong, L. M. Novak, and R. T. Shin, "Optimal polarizations for achieving maximal constrast in radar images,” J. Geophys. Res. 93, 15252-15260 (1988).

12. B. G. Hoover and J. S. Tyo, "Polarization components analysis for invariant discrimination," Appl. Opt. 46, 8364-8373 (2007).

13. M. Richert, X. Orlik, and A. De Martino, "Adapted polarization state contrast image," Opt. Express 17, 14199-14210 (2009).
14. F. Goudail, "Optimization of the contrast in active Stokes images," Opt. Lett. 34, 121-123 (2009).

15. J. S. Tyo, Z. Wang, S. J. Johnson, and B. Hoover, "Design and optimization of partial Mueller matrix polarizers," Appl. Opt. 49, 2326-2333 (2010).

16. D. Upadhyay, M. Richert, E. Lacot, A. D. Martino, and X. Orlik, "Effect of speckle on APSCI method and Mueller imaging," Opt. Express 19, 4553-4559 (2011).

17. G. Anna, F. Goudail, and D. Dolfi, "Polarimetric target detection in the presence of spatially fluctuating Mueller matrices," Opt. Lett. 36, 4590-4592 (2011).

18. S. Y. Lu and R. A. Chipman, "Interpretation of Mueller matrices based on polar decomposition," J. Opt. Soc. Am. A 13, 1106-1113 (1996).

19. R. Ossikovski, "Interpretation of nondepolarizing Mueller matrices based on singular-value decomposition," J. Opt. Soc. Am. A 25, 473-482 (2008).

20. F. Goudail and P. Réfrégier, "Statistical techniques for target detection in polarisation diversity images," Opt. Lett. 26, 644-646 (2001).

21. S. Breugnot and P. Clémenceau, "Modeling and performances of a polarization active imager at $\lambda=806 \mathrm{~nm}$," Opt. Eng. 39, 2681-2688 (2000).

22. S. L. Jacques, J. C. Ramella-Roman, and K. Lee, "Imaging skin pathology with polarized light," J. Biomed. Opt. 7, 329-340 (2002).

23. A. Bénière, F. Goudail, M. Alouini, and D. Dolfi, "Design and experimental validation of a snapshot polarization contrast imager," Appl. Opt. 48, 5764-5773 (2009).

24. A. Bénière, F. Goudail, M. Alouini, and D. Dolfi, "Precision of degree of polarization estimation in the presence of additive Gaussian detector noise," Opt. Commun. 278, 264-269 (2007).

25. F. Goudail and A. Bénière, "Optimization of the contrast in polarimetric scalar images," Opt. Lett. 34, 1471-1473 (2009).

26. F. Goudail and J. S. Tyo, "When is polarimetric imaging preferable to intensity imaging for target detection?" J. Opt. Soc. Am. A 28, 46-53 (2011).

27. F. Goudail, "Comparison of the maximal achievable contrast in scalar, Stokes and Mueller images," Opt. Lett. 35, 2600-2602 (2010).

28. H. D. Noble and R. A. Chipman, "Mueller matrix roots algorithm and computational considerations," Opt. Express 20, 17-31 (2012).

29. A. Bénière, F. Goudail, M. Alouini, and D. Dolfi, "Estimation precision of degree of polarization in the presence of signaldependent and additive Poisson noises," J. Eur. Opt. Soc. Rapid Publ. 3, 08002 (2008).

30. F. Goudail, P. Réfrégier, and G. Delyon, "Bhattacharyya distance as a contrast parameter for statistical processing of noisy optical images," J. Opt. Soc. Am. A 21, 1231-1240 (2004)

31. Q. Y. Duan, V. K. Gupta, and S. Sorooshian, "A shuffled complex evolution approach for effective and efficient global minimization,” J. Optim. Theory Appl. 76, 501-521 (1993).

32. G. Anna, F. Goudail, and D. Dolfi, "Optimal discrimination of multiple regions with an active polarimetric imager," Opt. Express 19, 25367-25378 (2011) 